\title{
Establishment of an effective nursing team against COVID-19-a COVID-19 treatment center experience
}

\author{
Xiaoxiao Li, Xiaohan $\mathbf{Q u}^{\wedge}$ \\ Department of Thoracic Surgery, The First Hospital of China Medical University, Shenyang, China \\ Contributions: (I) Conception and design: X Li; (II) Administrative support: X Qu; (III) Provision of study materials or patients: X Li; (IV) Collection \\ and assembly of data: Both authors; (V) Data analysis and interpretation: Both authors; (VI) Manuscript writing: Both authors; (VII) Final approval of \\ manuscript: Both authors. \\ Correspondence to: Xiaohan Qu. Department of Thoracic Surgery, The First Hospital of China Medical University, No.155 Nanjing North Street, \\ Heping District, Shenyang 110001, China. Email: han_seal@163.com.
}

\begin{abstract}
Background Novel coronavirus disease 2019 (COVID-19) is a serious infectious respiratory disease widespread worldwide. Nurses are the front-line staff in contact with infected patients and play a key role in treating patients and controlling the epidemic. The purpose of this study was to share our nursing team's experience in the treatment of COVID-19 and provide clinical guidance.

Methods: Detail nursing system arrangement was laid down: (I) reasonable division of ward channel was built; (II) effectively arranged human nursing resources and establishing special groups, including Training group, Critical patients nursing group, Quality control group, Epidemic preventive measures group, and Logistics support group; (III) optimize nursing workflow and establish various rules and regulations; (IV) scientific scheduling and humanized management; (V) pay attention to psychological support and adopt humanized management. The pre-job preparation, treatment results, and medical staff infection number were recorded.

Results: Fifty-four intensive care nurses all passed the training with an average score of $99.75 \pm 0.13$. One patient was dead, and 22 patients were discharged smoothly. The average length of stay was 9.12 days. The medical staff was not infected.

Conclusions: The treatment center was set up and functioning rapidly, safely, and orderly by implementing an emergency management strategy. The goal of a high rescue rate, low mortality, and no medical staff infection was achieved. This nursing system could be applied in COVID-19 patient treatment.
\end{abstract}

Keywords: Coronavirus disease 2019 (COVID-19); negative pressure isolation ward; public health emergency; emergency management

Submitted Apr 14, 2021. Accepted for publication Jun 08, 2021.

doi: 10.21037/apm-21-1255

View this article at: https://dx.doi.org/10.21037/apm-21-1255

\section{Introduction}

Novel coronavirus disease 2019 (COVID-19) was first reported in Hubei province, China, on December 31, 2019 (1). The novel coronavirus was named 2019 novel coronavirus (2019-nCoV) or the severe acute respiratory syndrome coronavirus 2 (SARS-CoV-2) by the World Health Organization (WHO) on January 12, 2020 (2). COVID-19 spread throughout the country during the Spring Festival rush in January. This highly contagious disease is a huge disaster for cities, countries and the world. At the time of writing, COVID-19 has killed over 590,000 people in the United States. In the battle against the COVID-19, the medical personnel were the frontline soldiers. Thus, well training and effective organization experiences of medical

\footnotetext{
$\wedge$ ORCID: 0000-0003-1494-2333.
} 
staffs is of great importance to fight against COVID-19. In one study from Taiwan, China, they reported creating and maintaining a good nursing working environment is important to attract more talented young people to take up nursing [Chen, 2020 \#7969]. However, they didn't report any effective nursing and organization experience. New cases occurred in Liaoning province at the same period in the January, 2020. To treat the COVID-19 patients promptly and effectively and prevent cross-infection or spread through the hospital, the Liaoning Provincial COVID-19 Centralized Treatment Center was set up quickly in the Jinzhou City Infectious Disease Hospital, according to the instructions of the Liaoning Provincial Health Commission. The author of this article served as the head of the nursing team and one of the directors of this treatment center from February to March 2020. We did an excellent job of treating COVID-19 infected patients. Our effective nursing experience can serve as a reference for nurses around the world. Thus, we reported the nursing emergency management measures in this article.

\section{Methods}

\section{Patient and nurse information}

Twenty-three patients in Shenyang, China were infected with COIVD-19 (13 men and 11 women, mean age \pm SD: $59.6 \pm 8.4$ years), treated in the Liaoning Provincial COVID-19 Centralized Treatment Center, and included in this study. The patients were transferred to our center after diagnosis. The diagnosis was according to the Protocol for diagnosis and treatment of COVID-19 that released by the National Health Commission of China.

The nurses (mean age \pm SD: $30.2 \pm 5.7$ years) with over ten years of working experience were from Liaoning Provincial COVID-19 Centralized Treatment Center.

\section{Ethical statement}

The study was conducted following the Declaration of Helsinki (as revised in 2013). The ethics board of the First Hospital of China Medical University approved the study (NO.: 20200116), and individual consent for this retrospective analysis was waived.

\section{Reasonable division of ward channel}

Reasonable and accurate division of ward channels can help quickly and orderly transport patients (3) and avoid infection of medical staff. The ward channel division is briefly summarized below.

\section{Rational distribution}

The ICU was designed as "three areas, two buffers, and four accesses". As "Three areas" implied, the ICU ward was divided into a clean area, a semi-contaminated area, and a contaminated area. Each area had a passing-by clothing room at the gate. "Two buffer rooms" refer to a buffer room with a flowing water-washing facility between the two areas. "Four channels" refers to two separated cleaning and contaminated areas of access for medical staff, and two separated cleaning and contaminated areas of access for patients were set up in the ward (Figure 1).

\section{Rehearsal and verification of rational distribution}

After 20 hours of preparation, the ward conducted a trial operation with repeated verification of the rationality and safety of the layout. The patient channel was used to simulate the transfer channel of patients in other cities, patient inspection channel, general transfer channel, re-transfer channel, dead patient transfer channel, and discharge channel. Medical channel: there are 18 access channels, including direct access to three areas, the evacuation channel of semi-polluted and polluted areas, the delivery channel of clean areas, the Waste Transportation channel, the delivery channel of drugs and articles. This channel system ensures the safety of patients and medical staff.

\section{Clearly labeled signs}

After confirming that the passageway was safe, a draft of the layout was shaped into a topographic map. Before the patients were transferred and stationed, each support unit started to conduct on-the-spot procedures and became familiar with the layout of "three areas, two buffers, and four accesses". All areas of access were marked with large characters to facilitate correct access and exit.

\section{Negative pressure isolation ward}

The negative pressure isolation ward uses a negative pressure environment (relative to the outside) in the ward to prevent the air containing pathogenic pathogens in the ward from diffusing to the outside after efficient filtration to achieve the safety aspect (4). The negative pressure isolation ward was mainly set up to deal with public health emergencies and major infectious diseases. It can supply a safe working environment for medical staff, 


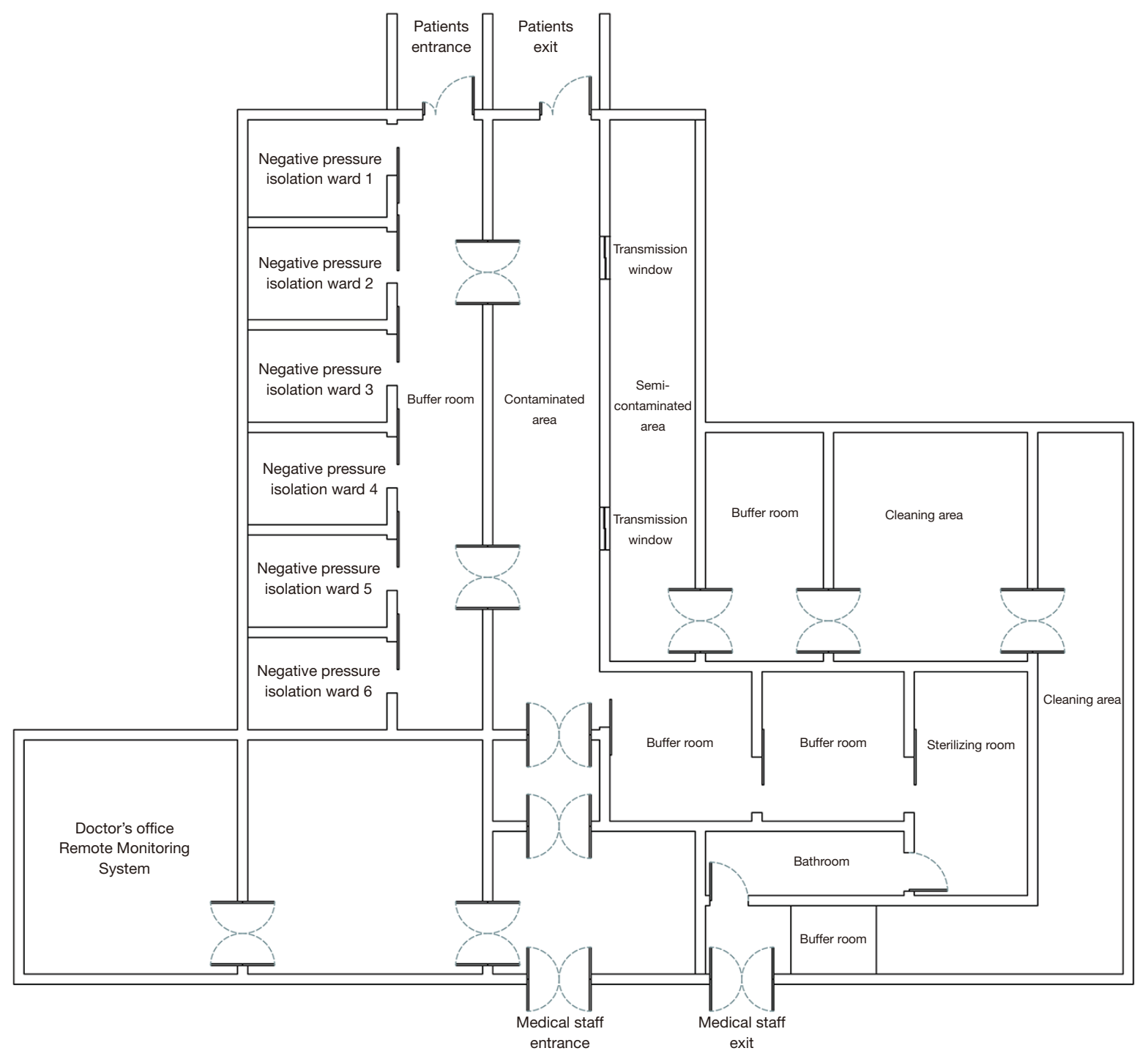

Figure 1 The structural design of the ICU ward.

a convenient and humanized medical place for patients, and an environment-friendly infectious disease hospital for society from the aspect of hardware facilities. Medical staff implemented all disinfection and isolation systems to reduce the number of irrelevant personnel entering and returning and strengthen monitoring to avoid infection in the hospital.

\section{Assignment of nursing human resources and establishing special groups}

Five special groups were quickly established to continuously improve epidemic treatment, namely the training group, the critical patients nursing group, the quality control group, the epidemic preventive measures group, and the logistics support group. The rescue center was rapidly formed through the cooperation between the groups (Figure 2).

\section{Training group}

\section{Putting on and taking off isolation gowns}

The training personnel assigned by the Liaoning Health Commission trained 70 medical staff personnel in our team to learn the proper procedure for putting on and taking off isolation gowns. Seventy people were divided into 7 groups of nurses. The training personnel examined each group leader after training, and then the group leader trained and 


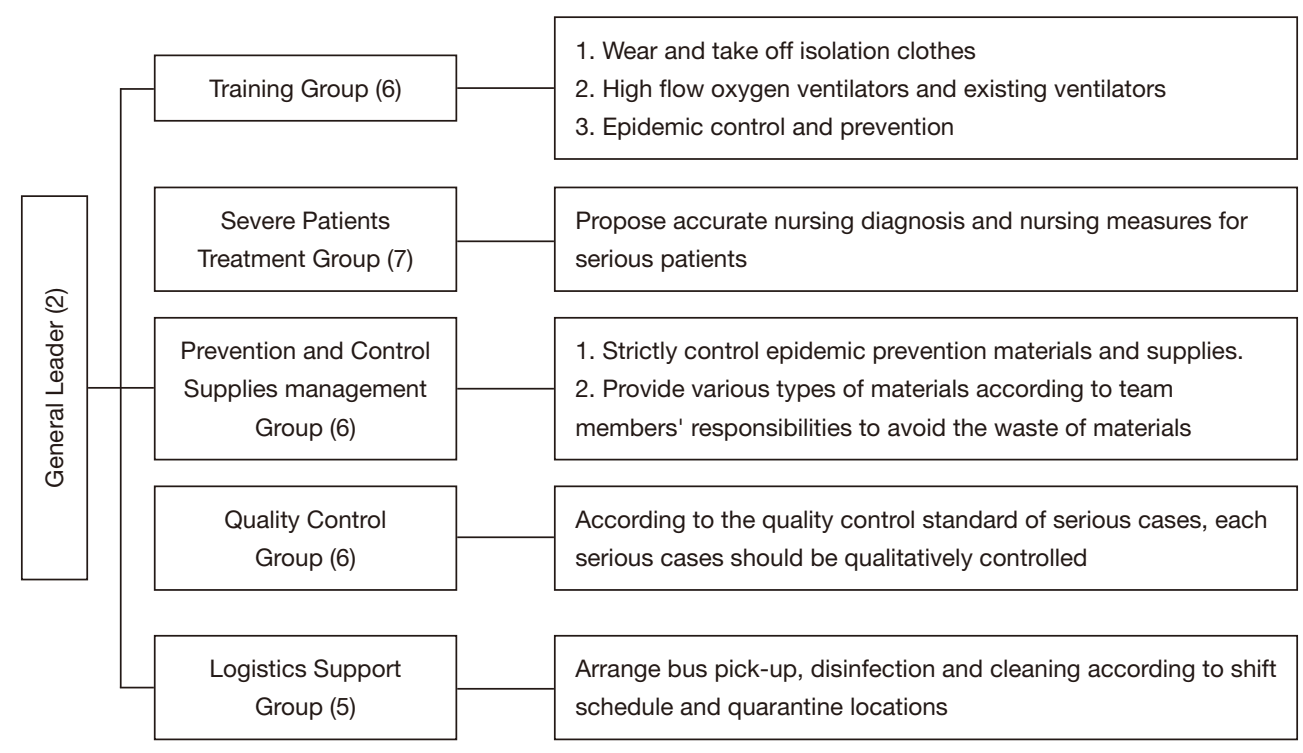

Figure 2 Assignment of nursing human resources and establishing special groups (5-7).

examined the staff one by one, thereby ensuring that every member of each group had an accurate comprehension of the process of putting on and taking off isolation gowns and emergency response methods.

\section{Training for the use of three types of respirators}

Three types of respirators were supplied to the ward. We contacted the equipment manufacturers, adopted video teaching modes, set up the pipeline connection, working mode, precautions, and alarm response measures to the group leaders' WeChat. Fifty-four nurses from different hospitals in the province were divided into 5 groups according to their qualifications, tenure, professional expertise, and whether they were in the ICU of their original unit or not). After training, each group leader trained and evaluated their group members. Additionally, the hospital invited infection control experts to supervise the nursing team's infection prevention and control measures.

\section{Critical patients nursing group}

Five critical care nursing groups were set up, as mentioned above. Each group serviced one negative pressure isolation room. The head nurse delivered a clinical nursing assessment of the patient according to the nursing duty report. The nursing diagnosis and nursing measures were conducted according to the vital signs and symptoms of each patient. Nursing measures were implemented according to the Prevention and Control Protocol for
COVID-19 released by the National Health Commission of the People's Republic of China.

\section{Quality control group}

The five group leaders of each group checked the quality of the contaminated area and semi-polluted area during the duty period on the premise of saving isolation gowns daily. The head nurse conducted the clinical firstlevel quality control, including assessment of critical patients, basic nursing, writing of a nursing record sheet, completing various forms, inpatient education, disinfection and isolation, preparation and application of emergent medicines, ward management, and clinical operation, and evaluation of the quality control results daily.

\section{Epidemic preventive measures group}

The provincial and municipal materials' management office specially supplied epidemic preventive equipment, including isolation gowns, N95 masks, shoe covers, sterile gloves, etc. However, because the quantity of the materials was uncertain, it was especially important to strictly manage using the preventive equipment.

(I) The duty list of medical staff was determined daily according to the patient's condition, and the isolation and protection articles were prepared according to body size to minimize the flow of personnel.

(II) Prepare the preventive equipment for the standby 
personnel for emergency use.

(III) Adjust the in and out of the process of sterilization personnel according to the workload to limit consumption of the preventive equipment to the greatest extent.

(IV) Daily reporting of the quantity of the preventive equipment and creating the supplying plan according to clinical needs.

\section{Logistics support group}

Fifty-four ICU nurses stayed in three different isolation hotels, six shifts every 24 hours, and the entire process of individual washing after taking off the isolation clothes took an average of 1 hour and 10 minutes after each shift. The next group team arrived at the cleaning area 30 minutes in advance for dressing. The bus frequency was at least 36 times daily. The arrangement of shifts for pick-up ensured that the medical staff had adequate rest and driving safety to the maximum extent.

\section{Optimize nursing workflow and establish various rules and regulations}

Due to the urgency of setting up a temporary ICU ward and the complexity of personnel composition, it was necessary to establish a series of scientific, effective, and practical working systems and processes to accomplish the institutionalization, standardization, and specialization of nursing functions to improve the success rate of treatment and avoid infection of medical staff.

\section{Formulate work responsibilities and post responsibility system}

According to the patient's condition and the Prevention and Control Protocol for COVID-19 released by the National Health Commission, we formulated The New Crown Severe ICU Nurses' Post Responsibility Systems, The New Crown Severe ICU Nurses Duties, and The New Crown Severe ICU Cleaning Staff Post Responsibility Systems to ensure the efficient and accurate operation of nursing functions.

\section{Develop operating processes to ensure safe, efficient, and orderly work}

To ensure the safety of the medical staff, we developed COVID-19 ICU Staffs Shift guideline, COVID-19 ICU
Nurses Operating Process, ICU Staffs Occupational Exposure Emergency Process, as well as other processes including Terminal Disinfection Process, Specimen Delivery Process, Death Transportation Process, and the patient's excrement disposal Process to keep the ward operating safely and efficiently.

\section{The perfect system to guarantee nursing quality}

To guarantee nursing quality for ICU patients, as many as 13 nursing management systems were set up, including Workflow of ICU Staffs, Order Implementation System, Nursing Discussion of Dead Cases System, etc.

\section{Scientific scheduling and humanized management}

\section{Basic information and situation of nursing staff}

The nursing staff of the COVID-19 ICU was recruited from several hospitals in Liaoning province; some were from intensive care units, while others were from general wards. They came to the COVID-19 centralized treatment center for temporary service. The nursing duties were divided according to the tenure of each nursing staff member, the level of experience in critical care, the level of the unit where they worked, the administrative position, whether they had undertaken the work of infection control or nursing quality control in their parent hospitals, etc., to make use of their strengths and improve work efficiency.

\section{Reasonable and flexible scheduling to avoid risks}

Due to the characteristics of the negative pressure isolation ward, nurses could not adapt to the environment within an adequate amount of time. They needed to deal with emergencies at any given time to ensure the operation of the nursing functions for the critical patients. In addition to nursing duties, nursing staff also had to undertake other work, which could cause physical exertion and reduce their immunity. Therefore, it was more appropriate to adjust the nurse-to-patient ratio according to the actual nursing workload so that the nurse-to-patient ratio was no less than 3:1.

\section{Pay attention to psychological support and adopt bumanized management}

Attach importance to dynamic psychological evaluation and psychologically support the nurses

Most of the 54 nurses in the treatment center did not participate in the hospital emergency medical team; they 
did not receive special nursing training for infectious diseases or emergency treatment training for public health events. There was a significant age difference among the nursing team members. In the early stage of the epidemic, unexpected events, including unfamiliar surroundings, arduous tasks, physical fatigue, and emergency mobilization, led to psychological disorders in most of the nurses. The leader was aware of each nurse's psychological state and provided psychological interventions for the nurses with signs of depression at first notice. Additionally, humanistic care and psychological counseling should be carried out, including additional salary allowances, a guarantee of living materials, humanized shift arrangements, and public reporting of work experiences, to help nurses adjust their emotions to ensure that they can complete their work in a satisfactory psychological state (5).

\section{Attach importance to the psychological reaction of patients and adopt humanized management}

Most of the patients transferred to the ICU were migrant workers returning from the coronavirus epicenter Wuhan. Most were part of a family outbreak. Family members were scattered over various areas due to different conditions. Some patients had various levels of anxiety or depression because they were concerned about their families. In addition to conventional nursing, the primary nurses should conduct a psychological nursing intervention for patients. Psychological nursing intervention includes (I) using the video telephone, intercom call, or conducting observation and waving to reduce the anxiety of patients; (II) playing light music and administering warming Chinese medicine to adjust patients' mentality; (III) serving food according to the patient's preference to ensure their nutritional status and improve their immunity.

\section{Statistical analysis}

The data were recorded with EXCEL and measurement data were presented as mean \pm standard deviation.

\section{Results}

\section{Pre-work training}

Because the time is appropriate and the task is heavy, we were required to complete the training of 54 intensive care nurses. Four group leaders led each group of 10 people and conducted five steps of teaching, practice, discussion, practice, and assessment in batches. We were required to master the details of putting on and taking off protective equipment one by one at each step. We focused on the training of taking off protective clothing to ensure zero infection rate of medical staff. According to the operating standard of wearing and taking off protective clothing, nurses with a score of 98 or higher were allowed to work. The average score of 54 intensive nurses was $99.75 \pm 0.13$. All staff met the standards.

\section{The results of treatment}

The task of Jinzhou Treatment Center in Liaoning Province lasted for 32 days. Nursing staff work in the negative pressure ward for 4 hours out of every 24 hours. During the 32-day mission, 23 patients with COVID-19 infection were received, among which one died, one was in critical type, five were in severe type, and 16 were in normal type. The average length of stay was 9.12 days, and all 22 patients were cured and discharged. The medical staff was not infected.

\section{Discussion}

COVID-19 is firstly diagnosed in Wuhan, China, on December 31, 2019 (6). On March 11, 2020, WHO declared COVID-19 was a severe global pandemic disease. It has spread so rapidly, it has caused outbreaks in virtually every country globally (7), as there is currently no effective treatment. The main treatment is symptomatic and supportive treatment, which includes maintenance of vital signs, oxygen saturation, and blood pressure.

Further, treatment of complications, including secondary infections or organ failure, should be conducted. As the first-line care provider of inpatients, nurses play an important role in treating patients and preventing the spread of diseases (8). Thus, strengthening the professional ability and epidemic prevention capacity of nursing staff is conducive to better patient treatment and prevention of medical staff infection and further spread of infection, which is crucial to controlling the epidemic.

Scientific care is a conception composed of scientific scheduling, scientific organization and scientific management. The care of COVID-19 is very different from the care of conventional infectious diseases. Nurses need to be better equipped and more aware of the requirement for anti-infection. Scientific care can provide more comprehensive training about COVID-19 infection and spread, better plan the staff flow and material allocation 
of the care facility, pay more attention to the physical and mental health of the nurses, and prevent the physical and psychological problems of the nurses. In this way, scientific care could be an efficient way to prevent COVID-19 infection and treat COVID-19 patients.

Earlier studies indicated that nursing of serious infectious respiratory diseases, like the Middle East respiratory syndrome (MERS), was grim. The nurses could experience anxiety and have psychological problems due to an unprepared therapeutic environment, a burdensome care process, and a risk of infection (9). Besides, in one H1N1 study, nurses would encounter many challenges, like personal protective equipment, infection control procedures, fear of infection and transmitted diseases, new roles for staff, morale levels, and complex training and challenges (10). Mental health problems will be induced in nurses in the process of fighting against a new infectious disease. Huang et al. confirmed that the medical staff could suffer anxiety and stress during the fighting against COVID-19 (11). Thus, reducing the negative effect of heavy anti-COVID-19 work on the nurses will greatly benefit epidemic prevention.

The protective equipment don't allow the nurse to drink water or go to the toilet frequently. For convenience, nurses often go to work without food or drink until the end of their shift. As a result, multiple shifts are needed to complete $24 \mathrm{~h}$ patient care. In this way, the physical and mental health of the nurse could be guaranteed.

In this study, to satisfactorily deal with the adverse factors encountered in nursing work, we established an effective nursing team to treat COVID-19 patients. This team was well organized, and an in-detail description of this nursing system was shown as follows: (I) reasonable division of ward channel was built; (II) effectively arranged nursing human resources and establishing special groups, including training group, critical patients nursing group, quality control group, epidemiological preventive measures group, and logistics support group; (III) optimize nursing workflow and establish various rules and regulations; (IV) scientific scheduling and humanized management; (V) pay attention to psychological support and adopt humanized management. Under the management of this nursing system, all the nurses completed the pre-job training. Except for the death of 1 critical patient, all the other patients were discharged smoothly. The patient developed acute respiratory distress syndrome, which, with aggressive treatment, worsened, progressed to respiratory failure and eventually death. No infection occurred to all the medical staff, and all the nurses were mentally healthy.

\section{Conclusions}

When confronted with a sudden outbreak of public health events, both clinical medical staff and managers lacked sufficient experience. We summarized our experiences in response to this epidemic, including the establishment of an emergent nursing team, integration of multiple components of the nursing team, the coordinated operation of each part, scientific organization, and dynamic, flexible scheduling, formulation of the reasonable working processes and systems, optimization of the treatment model, and evaluation of the medical staff and patients' psychological conditions. Thanks to the implementation of an emergency management strategy, the treatment center achieved the goal of a high rescue rate, low mortality, and absence of infection to medical staff. The nursing task of the Liaoning provincial COVID-19 treatment center succeeded in nursing care of the patients with COVID-19.

\section{Acknowledgments}

The authors thank Professor Bin Shan from Elson S. Floyd College of Medicine, Washington State UniversitySpokane, for language help and proofreading.

Funding: This work was supported by the Doctoral Startup Foundation of Liaoning Province (grant number 20170520349).

\section{Footnote}

Data Sharing Statement: Available at https://dx.doi. org/10.21037/apm-21-1255

Conflicts of Interest: Both authors have completed the ICMJE uniform disclosure form (available at https://dx.doi. org/10.21037/apm-21-1255). Both authors have no conflicts of interest to declare.

Etbical Statement: The authors are accountable for all aspects of the work in ensuring that questions related to the accuracy or integrity of any part of the work are appropriately investigated and resolved. The study was conducted following the Declaration of Helsinki (as revised in 2013). The ethics board of the First Hospital of China Medical University approved the study (NO.:20200116), and individual consent for this retrospective analysis was waived. 
Open Access Statement: This is an Open Access article distributed in accordance with the Creative Commons Attribution-NonCommercial-NoDerivs 4.0 International License (CC BY-NC-ND 4.0), which permits the noncommercial replication and distribution of the article with the strict proviso that no changes or edits are made and the original work is properly cited (including links to both the formal publication through the relevant DOI and the license). See: https://creativecommons.org/licenses/by-nc-nd/4.0/.

\section{References}

1. Zhou P, Yang XL, Wang XG, et al. A pneumonia outbreak associated with a new coronavirus of probable bat origin. Nature 2020;579:270-3.

2. Wang T, Du Z, Zhu F, et al. Comorbidities and multiorgan injuries in the treatment of COVID-19. Lancet 2020;395:e52.

3. Reeves K. Patient transportation. J Emerg Nurs 2010;36:302; author reply 302.

4. Miller SL, Clements N, Elliott SA, et al. Implementing a negative-pressure isolation ward for a surge in airborne infectious patients. Am J Infect Control 2017;45:652-9.

5. Arons MM, Hatfield KM, Reddy SC, et al. Presymptomatic SARS-CoV-2 Infections and Transmission in a Skilled

Cite this article as: $\mathrm{Li} \mathrm{X,} \mathrm{Qu} \mathrm{X.} \mathrm{Establishment} \mathrm{of} \mathrm{an} \mathrm{effective}$ nursing team against COVID-19-a COVID-19 treatment center experience. Ann Palliat Med 2021;10(6):6661-6668. doi: 10.21037/apm-21-1255
Nursing Facility. N Engl J Med 2020;382:2081-90.

6. Munster VJ, Koopmans M, van Doremalen N, et al. A Novel Coronavirus Emerging in China - Key Questions for Impact Assessment. N Engl J Med 2020;382:692-4.

7. Sadati AK, B Lankarani MH, Bagheri Lankarani K. Risk Society, Global Vulnerability and Fragile Resilience; Sociological View on the Coronavirus Outbreak. Shiraz E-Med J 2020;21:e102263.

8. Barnett DJ, Thompson CB, Errett NA, et al. Determinants of emergency response willingness in the local public health workforce by jurisdictional and scenario patterns: a cross-sectional survey. BMC Public Health 2012;12:164.

9. Kim Y. Nurses' experiences of care for patients with Middle East respiratory syndrome-coronavirus in South Korea. Am J Infect Control 2018;46:781-7.

10. Corley A, Hammond NE, Fraser JF. The experiences of health care workers employed in an Australian intensive care unit during the H1N1 Influenza pandemic of 2009: a phenomenological study. Int J Nurs Stud 2010;47:577-85.

11. Huang JZ, Han MF, Luo TD, et al. Mental health survey of medical staff in a tertiary infectious disease hospital for COVID-19. Zhonghua Lao Dong Wei Sheng Zhi Ye Bing Za Zhi 2020;38:192-5.

(English Language Editor: J. Chapnick) 